\title{
On the Lifetime Performances of Dielectric Elastomer Transducers Under Constant Electric-Stress Loading
}

\author{
Yi Chen*, Lorenzo Agostini**, Marco Fontana***, Rocco Vertechy* \\ * Department of Industrial Engineering, University of Bologna \\ Bologna, Italy(e-mail:yi.chen4@unibo.it,rocco.vertechy@unibo.it) \\ ** PERCRO Laboratory - TeCIP Institute, Scuola Superiore Sant'Anna \\ Pisa, Italy (e-mail: lorenzo.agostini@santannapisa.it) \\ *** Department of Industrial Engineering, University of Trento, \\ Trento, Italy, (e-mail: marco.fontana-2@unitn.it)
}

Keywords: Dielectric elastomer generators, Fatigue, Reliability, Weibull probability distribution.

\section{INTRODUCTION}

Dielectric elastomer transducers (DETs) are deformable capacitors, made by highly elastic dielectric layers coated with compliant electrodes. They are electrostatic devices that make it possible to convert mechanical energy into direct current electricity and vice-versa (Carpi et al. (2008)). As such, they can be used to conceive solid-state electrostatic actuators, generators and sensors exhibiting the following properties (Carpi et al. (2008)): large energy and power densities; ease of manufacture and integration; good resistance to shocks and corrosion; silent operation; low cost.

Recently, commercial rubber membranes made of silicone elastomers, natural rubber and styrenic rubber demonstrated excellent electromechanical properties for the development of high energy density DETs. In particular, in experimental applications as generators, inflatable DETs based on silicone elastomers made it possible to consistently convert pneumatic energy into electricity at an energy density per cycle greater than $150 \mathrm{~J} / \mathrm{kg}$ (see for instance Moretti et al. (2017)) and greater than $400 \mathrm{~J} / \mathrm{kg}$ in case of styrenic rubber.

These experimented performances can however be sustained for a limited number of cycles only, after which the DET fails irreversibly. To date, very little information is available on the fatigue life performances of dielectric elastomer materials and of the transducers made thereof (Kornbluh et al. (2010)) Having identified electrical breakdown as the most probable mode of DET failure (Kornbluh et al. (2010)), this work reports and discusses on a set of lifetime constant electricstress tests conducted on frame stretched circular DET specimens made of a styrenic rubber.

\section{EXPERIMENTAL}

An experimental test-bench (see Fig. 1) has been purposely developed for the simultaneous testing of a batch of up to 16 DET specimens by subjecting them to cyclic loading with general electric field waveforms and no controlled force or displacement. The test-bench features fully automated operation and data acquisition/saving, including automatic detection and isolation of broken DETs.
Three different batches, each made of 16 frame-stretched circular DET specimens, were prepared using a custom made styrenic rubber as elastic dielectric, carbon grease as compliant electrodes and a Delrin holed plate as a clamping frame (see Fig. 2). All specimens were identical: $25 \mathrm{~mm}$ in diameter $\phi, 27 \mu \mathrm{m}$ in thickness $t$ and with 2.6 pre-stretch.

The developed experimental test-bench was used to perform lifetime constant-stress tests on the prepared specimen batches by subjecting them to square wave electric field signals with $50 \%$ duty-cycle, $1 \mathrm{~Hz}$ frequency and with the following amplitudes: $127 \mathrm{MV} / \mathrm{m}$ for the first batch, 100 $\mathrm{MV} / \mathrm{m}$ for the second batch and $70.3 \mathrm{MV} / \mathrm{m}$ for the third batch.

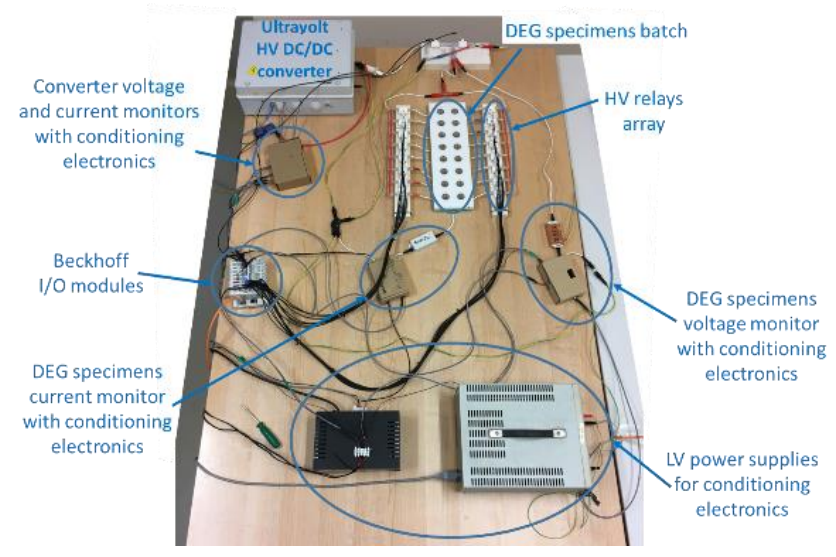

Fig. 1. Experimental set-up used for lifetime characterization.
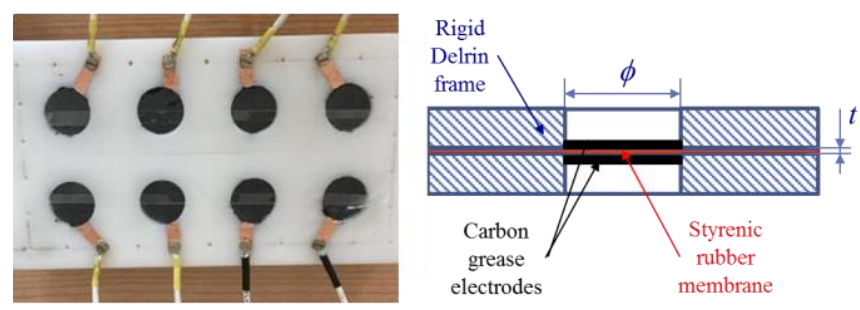

Fig. 2. Frame-stretched circular DET specimens: 1 batch of 8 specimens during test (on the left), schematic of one specimen (on the right). 


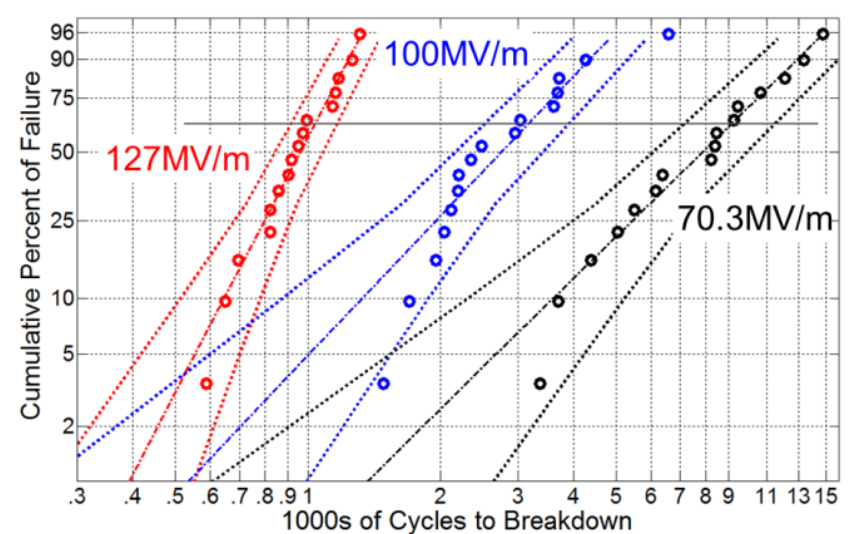

Fig. 3. Weibull plot of lifetime for specimen batches tested at different electric field levels.

Obtained results showed that identical specimens tested at the same level of applied electric field fail at different numbers of cycles, with a rather wide spread of the data. This indicated that electrical breakdown of DETs is a stochastic process.

Acquired data were then manipulated via the Ross's method (as described in the standard IEC 62539) to calculate the probability of failure associated to each broken specimen. Results are shown in Fig. 3: red circles for the batch tested at $127 \mathrm{MV} / \mathrm{m}$, blue circles for the batch tested at $100 \mathrm{MV} / \mathrm{m}$, black circles for the batch tested at $70.3 \mathrm{MV} / \mathrm{m}$.

\section{MODEL}

An analytical model was developed to correlate the number of cycles to failure to the time-law of application of electric field loads.

Following a practice existing in the power cables industry (see for instance Dissado and Fothergill (1992)), we have assumed that the time-to-breakdown dependency on applied electric field of a DET can be described by a Weibull probability distribution in the form:

$$
P_{F}(E, t)=1-\exp \left\{-c \int_{0}^{t} a t^{a-1}[E(t)]^{b} d t\right\}
$$

which, for square wave electric field signals becomes

$$
\begin{gathered}
P_{F}(E, N)=1-\exp \left\{\left(\frac{N}{N_{c}^{E}}\right)^{a}\right\}, N_{c}^{E}=N_{c}\left(\frac{E_{c}}{E}\right)^{\frac{b}{a}} \\
M C T F_{E}=N_{c}^{E} \cdot \Gamma(1+1 / a)
\end{gathered}
$$

where $a, b, E_{c}$ and $N_{c}$ are parameters to be found via numerical fitting of experimental data, while $E$ is the amplitude of the electric field signal. $N_{c}^{E}$ is called the characteristic life, whereas $M C T F_{E}$ is the mean cycle to failure.
The model was then fitted to the acquired experimental data. Results are shown in Fig. 3 with dash-dotted lines (dotted lines represent $90 \%$ confidence bounds).

\section{DISCUSSION}

Results highlight:

- a strong dependency of DET lifetime on the level of applied electric field; in particular, lifetime decreases as the electric field amplitude is increased;

- the proposed model is adequate to capture the correlation between level of electrical loading and failure in DETs;

- the custom made styrenic rubber shows a MCTF higher than 7000 cycles when loaded with an electric field amplitude of $70.3 \mathrm{MV} / \mathrm{m}$; this lifetime is larger than that reported in Kornbluh et al. (2010) for DET specimens based on acrylic elastomers (VHB 4910 by 3M) and subjected to the same electric field level.

\section{CONCLUSIONS}

This work described the approach, experimental set-up and procedures that have been developed to characterize the lifetime of dielectric elastomer transducers (DETs) against electrical loading. In addition, it reported and discussed the results obtained from the lifetime testing of frame-stretched circular DET specimens based on a custom-made styrenic rubber. The proposed methodology and obtained experimental results can be used in a design procedure to find optimal trade-offs between DET performance and lifetime/reliability.

Future work on lifetime characterization of DETs will consider: 1) effects of electrical loading frequency; 2) effects of specimen size; 3) effect of specimen pre-stretch; 4) other materials such as silicone elastomers; 5) mixed electromechanical loading.

\section{REFERENCES}

Carpi F., De Rossi D., Kornbluh R. (2008). Dielectric elastomers as electromechanical transducers: Fundamentals, materials, devices, models and applications of an emerging electroactive polymer technology. Elsevier Science, Amsterdam.

Dissado L.A. and Fothergill J.C. (1992). Electrical degradation and breakdown in polymers. No. 9., IET.

Kornbluh R.,Wong-Foy A., Pelrine R., Prahlad H. and McCoy B. (2010). Long-lifetime all-polymer artificial muscle transducers. MRS proceedings, vol. 1271. Cambridge University Press.

Moretti G., Righi M., Vertechy R.; Fontana M (2017). Fabrication and Test of an Inflated Circular Diaphragm Dielectric Elastomer Generator Based on PDMS Rubber Composite. Polymers, vol. 9, n. 7, p. 283. 